\title{
Analysis of the factors influencing the acetylation rate of wood
}

\begin{abstract}
Factors influencing the rate of acetylation were examined based on the swelling of wood in the reaction solution and the dimensions of the wood sample. The activation energy of acetylation was also estimated. In a swelling test, it was found that wood swells thoroughly in acetic anhydride even without pyridine above $60^{\circ} \mathrm{C}$. Therefore, pyridine may facilitate the acetylation process as a catalyst and not as a swelling agent. The weight gain, $x(\%)$, attained at reaction time $t(\mathrm{~h})$, for various compositions of acetylation solution or dimensions of wood sample were analyzed by applying an original rate equation $\left[x=a \times\left(1-\mathrm{e}^{-k t}\right)^{1 / n}\right]$, where $a$ is the ultimate weight gain (\%), $k$ is the rate constant $\left(\mathrm{h}^{-1}\right)$, and $n$ is a measure of the hindrance against the diffusion of reagent. The optimum volume fraction of pyridine in the pyridine-catalyzed acetylation was about 0.2. Accompanied by a rise in pyridine content, the reaction showed increased diffusion-controlled behavior. The rate constant, which is not affected by the dimensions of the wood sample, was estimated from which an activation energy of about $130 \mathrm{~kJ} / \mathrm{mol}$ was calculated.
\end{abstract}

Key words Acetylation $\cdot$ Reaction rate $\cdot$ Pyridine $\cdot$ Diffusion $\cdot$ Swelling

\section{Introduction}

The acetylation of wood has been studied for some time, and is one of the most practical methods of chemical modification of wood. Meanwhile, fundamental studies on the topic continue even now, which will contribute to the progressive application of acetylation.

K. Minato $(\bowtie) \cdot Y$. Ito

Graduate School of Agriculture, Kyoto Prefectural University,

Shimogamo Hangi-cho, Sakyo-ku, Kyoto 606-8522, Japan

Tel. +81-75-703-5646; Fax +81-75-703-5646

e-mail: minato@kpu.ac.jp
Studies by Hill et al. ${ }^{1}$ and Hill and Papadopoulos ${ }^{2}$ have focused on reaction kinetics, and have discussed the reaction mechanism. We have also investigated the reaction kinetics of wood acetylation, and while Hill et al. ${ }^{1}$ reported an activation energy of $20-50 \mathrm{~kJ} / \mathrm{mol}$, we $\mathrm{e}^{3}$ obtained a value greater than $100 \mathrm{~kJ} / \mathrm{mol}$. In that study, we attributed the apparent difference to the rate-determining step. Hill et al. ${ }^{1}$ used Corsican pine wood blocks $(5 \mathrm{~mm}$ in longitudinal direction), while we used wood meal of Sitka spruce (150 $355 \mu \mathrm{m}$ ). Although acetylation proceeds heterogeneously irrespective of the dimensions or shape of the wood, the accessibility of the reagent to the cell wall would differ depending on the size of the sample.

Concerning accessibility of the reagent, the reaction solution may also affect the swelling of wood. In the previous study, ${ }^{3}$ we carried out the acetylation in neat acetic anhydride, a xylene-acetic anhydride mixture (v/v, $1: 1$ and $1: 3$ ), and a pyridine-acetic anhydride mixture ( $\mathrm{v} / \mathrm{v}, 2: 8)$, and explained the difference in activation energy based on the degree of swelling in the reaction solution. In those cases, the rate-determining step was believed to shift depending on the type of reaction system.

In this study, we concentrated our attention on the swelling, namely the composition of the reaction solution, in the pyridine-acetic anhydride system and the dimensions of the wood sample. These two factors are closely concerned with the accessibility of the reagent to the reaction site in the cell wall. The mechanism of acetylation was analyzed using the reaction kinetics.

\section{Materials and methods}

Wood samples and reagents

Fine $(150-355 \mu \mathrm{m})$ and coarse $(355-850 \mu \mathrm{m})$ wood meal prepared by a Wiley mill, and sawed cross sections, $15(\mathrm{~T}) \times 20$ $(\mathrm{R}) \times 2 \mathrm{~mm}(\mathrm{~L})$ and $30(\mathrm{~T}) \times 30(\mathrm{R}) \times 5 \mathrm{~mm}(\mathrm{~L})$, were obtained from Sitka spruce (Picea sitchensis Carr.). All samples were treated with an ethanol-benzene mixture 
(v/v, 1:2) using a Soxhlet extractor for 6-8h. Reagent-grade acetic anhydride, pyridine, and $N, N$-dimethylformamide (DMF) were used.

Evaluation of the swelling in various solutions

The degree of swelling was determined in various mixtures of acetic anhydride and pyridine or DMF. The volume fractions of pyridine or DMF in the solution were $0,0.1,0.2,0.5$, 0.8 , and 1 . Five matching cross sections $(5 \mathrm{~mm})$ were assigned for each solution. The oven-dry specimens were immersed in the solutions at room temperature, and the flasks were evacuated with a water aspirator until all bubbling stopped. Dimensions in the radial and tangential directions were occasionally measured, and when they reached a stable value, the temperature was increased. The determinations were continued at $40^{\circ}, 60^{\circ}$, and $80^{\circ} \mathrm{C}$ using the same specimen. Relative swelling was calculated by dividing the volumetric swelling in each solution by that in pure water.

Measurement of reaction rate in various reaction mixtures

The reaction rate was determined in various solutions of pyridine-acetic anhydride and DMF-acetic anhydride. Wood meal of $150-355 \mu \mathrm{m}$ particle size was used. The procedures were the same as those described previously. ${ }^{3}$ The volume fraction of pyridine in the reaction system was varied from 0.05 to 0.8 while that of DMF was kept at 0.2 . The reactions were conducted at $100^{\circ} \mathrm{C}$.

Determination of reaction rate using different shaped wood samples

Wood meal of $355-850 \mu \mathrm{m}$ particle size and 2-mm-thick cross sections were used. The acetylation was carried out in the uncatalyzed system at $100^{\circ}-120^{\circ} \mathrm{C}$.

\section{Results and discussion}

Swelling of wood in various mixed solutions

The relative swelling of wood blocks in the pyridine-acetic anhydride mixtures is plotted against the volume fraction of pyridine in Fig. 1. Above $60^{\circ} \mathrm{C}$, the relative swelling was about 1.2 , although for neat pyridine it was slightly smaller. Below $40^{\circ} \mathrm{C}$, swelling was remarkably low, notably when the pyridine content was low. The pyridine-catalyzed acetylation is usually carried out with a pyridine volume fraction of 0.2 at which relative swelling is around 1.2 when above $60^{\circ} \mathrm{C}$, although the swelling is much less below $40^{\circ} \mathrm{C}$. Acetylation is sometimes conducted only with acetic anhydride, in which case the wood swells to the same degree except at impractical low temperatures. There may be a critical temperature for wood swelling in the range of $40^{\circ}-60^{\circ} \mathrm{C}$ that is related to the softening temperature of wood. ${ }^{4}$ At any rate, the low degree of swelling at low temperature is not im-

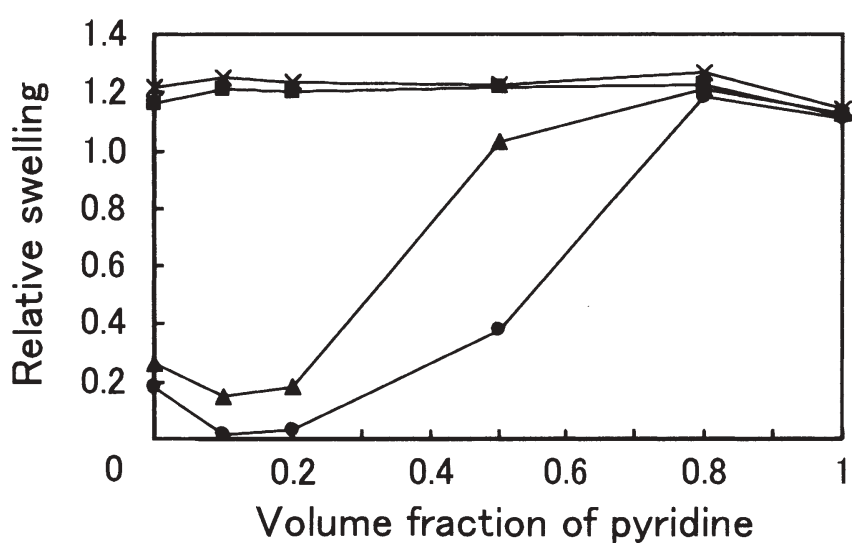

Fig. 1. Relative swelling of wood in the pyridine-acetic anhydride mixtures. Circles, room temperature; triangles, $40^{\circ} \mathrm{C}$; squares, $60^{\circ} \mathrm{C}$; crosses, $80^{\circ} \mathrm{C}$

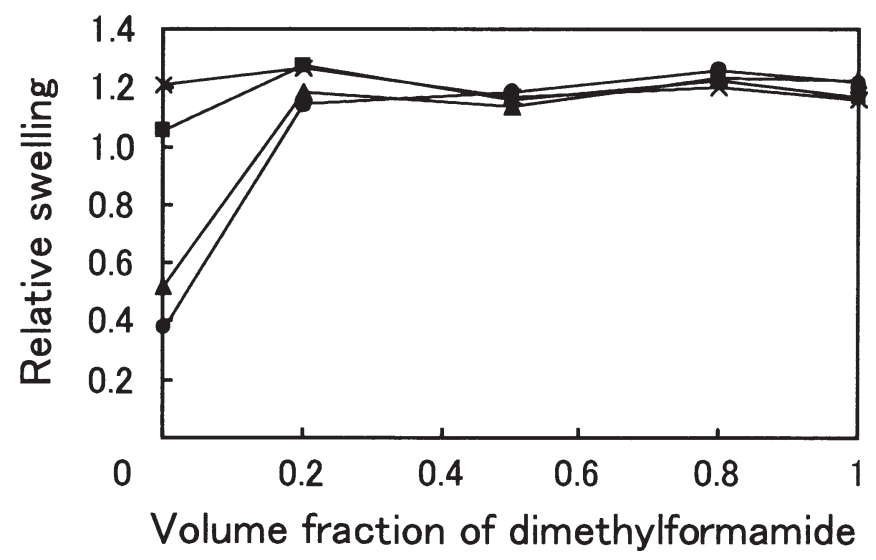

Fig. 2. Relative swelling of wood in the dimethylformamide-acetic anhydride mixtures. Symbols are the same as in Fig. 1

portant, because acetylation is not practical at such low temperatures.

We have reported that the reaction in the pyridine-acetic anhydride (v/v, 2:8) system is about 80 times faster than the uncatalyzed reaction at $80^{\circ} \mathrm{C}^{3}$ However, because relative swelling did not differ significantly between these systems, the difference in the reaction rate cannot be explained by the degree of swelling in the reaction solution. The major function of pyridine may be as a catalyst rather than as a swelling agent.

Figure 2 shows the result obtained for the DMF-acetic anhydride mixtures. The relative swelling reached 1.1-1.2 at volume fractions above 0.2 , even at room temperature. Nevertheless, as will be described later, the reaction rate in the DMF solution was no greater than that in pyridine solution. The difference between pyridine and DMF is also attributable to catalysis and not to swelling.

Dependence of reaction parameters on the composition of the reaction mixture

Figure 3 shows an example of a reaction profile in which weight gain is plotted against reaction time. Weight gain 
increased exponentially at first, and then leveled off at around $35 \%$. A rate equation in which diffusion of the reagent is taken into consideration was applied to the data. The equation, recently proposed by us, ${ }^{5}$ is

$x=a \times\left(1-\mathrm{e}^{-k t}\right)^{1 / n}$

where $x$ is weight gain (\%) at reaction time $t(\mathrm{~h}), a$ is ultimate weight gain (\%), $k$ is the rate constant $\left(\mathrm{h}^{-1}\right)$, and $n$ is a measure of the hindrance against the diffusion of reagent into the reaction site. When $n$ is larger than unity, the reaction is controlled by diffusion.

The reaction parameters and statistical data are summarized in Table 1 . The rate equation was successfully fitted to whole data, and the ratio of contribution, $R^{2}$, was higher than 0.95 for all reaction conditions. The curve drawn in Fig. 3 was obtained by regression with Eq. 1.

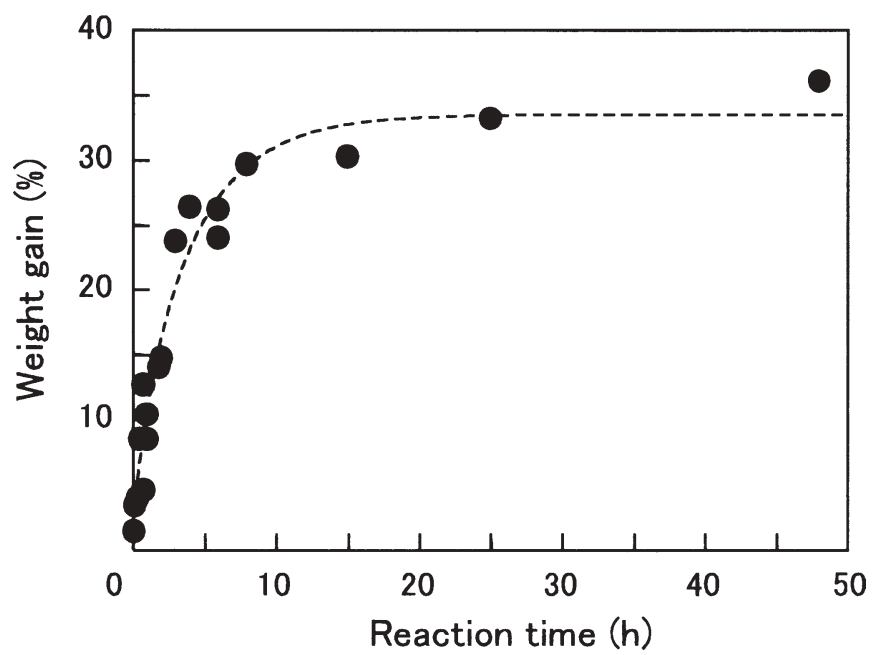

Fig. 3. An example of weight gain during reaction carried out in the pyridine-acetic anhydride mixture $(\mathrm{v} / \mathrm{v}, 0.5: 9.5)$ at $100^{\circ} \mathrm{C}$. Circles, observed value; dashed line, regression with Eq. 1
The calculated value of ultimate weight gain was around $33 \%$, irrespective of the volume fraction of pyridine. In contrast, $k$ increased with increasing pyridine content up to about 0.1 to 0.3 and then decreased (Fig. 4). The increase in $k$ may be attributable to the increase in the catalytic activity of pyridine, and the decrease after the peak may be due to the dilution of acetic anhydride. Parameter $n$ increased with a rise in pyridine content (Fig. 5). This means an intensification of the heterogeneity of the reaction accompanying the increase in the volume fraction of pyridine, i.e., an increase in catalysis. It has been reported that a volume fraction of 0.2 is optimal for pyridine-catalyzed acetylation, ${ }^{6}$ and this was confirmed by the reaction rate.

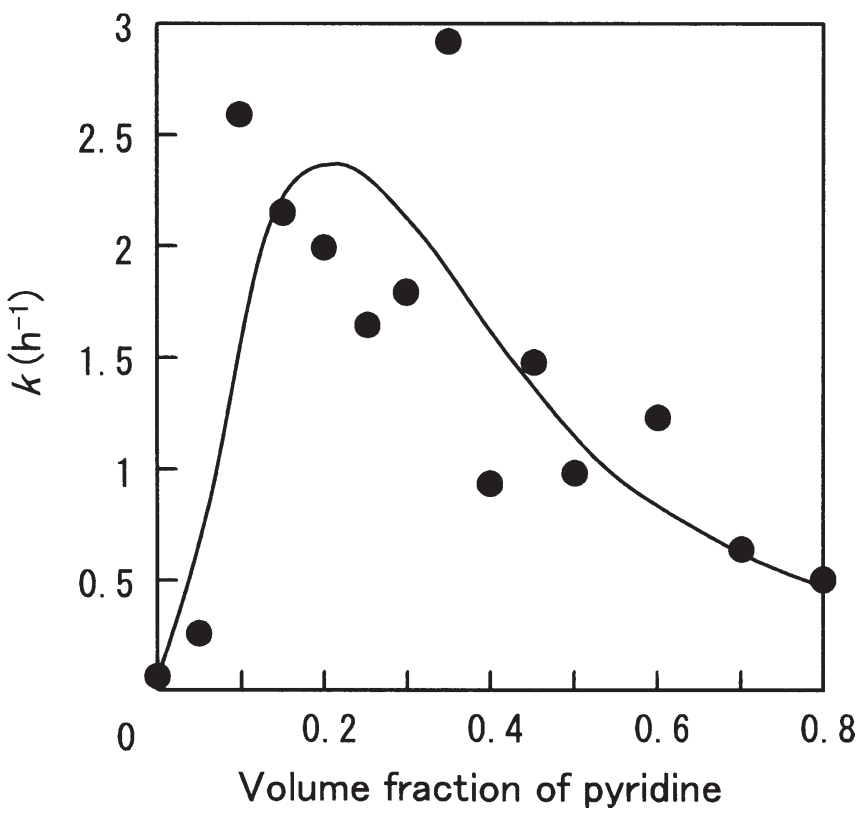

Fig. 4. Dependence of rate constant at $100^{\circ} \mathrm{C}$ on the volume fraction of pyridine in the pyridine-acetic anhydride mixtures

Table 1. Dependence of the acetylation reaction parameters on the volume fraction of pyridine or dimethylformamide (DMF) in the reaction solutions

\begin{tabular}{llllll}
\hline $\begin{array}{l}\text { Volume fraction of } \\
\text { pyridine (DMF) }\end{array}$ & $a(\%)$ & $k\left(\mathrm{~h}^{-1}\right)$ & $n$ & No. of data & $R^{2}$ \\
\hline $0^{\mathrm{a}}$ & 28.8 & $3.70 \times 10^{-2}$ & 1.25 & 14 & 0.990 \\
0.05 & 33.5 & $2.30 \times 10^{-1}$ & 1.36 & 18 & 0.958 \\
0.1 & 30.9 & 2.57 & 0.44 & 11 & 0.977 \\
0.15 & 30.5 & 2.13 & 0.88 & 13 & 0.976 \\
$0.2^{\mathrm{a}}$ & 28.5 & 1.97 & 1.51 & 8 & 0.966 \\
0.25 & 32.4 & 1.62 & 1.93 & 11 & 0.958 \\
0.3 & 32.2 & 1.76 & 2.25 & 9 & 0.963 \\
0.35 & 31.6 & 2.90 & 1.83 & 7 & 0.949 \\
0.4 & 34.7 & $9.05 \times 10^{-1}$ & 3.51 & 8 & 0.985 \\
0.45 & 33.7 & 1.45 & 3.47 & 7 & 0.966 \\
0.5 & 33.0 & $9.52 \times 10^{-1}$ & 3.83 & 8 & 0.999 \\
0.6 & 33.9 & 1.20 & 4.10 & 6 & 0.997 \\
0.7 & 32.4 & $6.09 \times 10^{-1}$ & 4.12 & 4 & 0.999 \\
0.8 & 32.7 & $4.73 \times 10^{-1}$ & 4.74 & 4 & 0.976 \\
$0.2)$ & 28.4 & $1.12 \times 10^{-1}$ & 1.90 & 13 & \\
\hline
\end{tabular}

The reaction was carried out using $150-355 \mu \mathrm{m}$ wood meal at $100^{\circ} \mathrm{C}$

${ }^{\mathrm{a}}$ Data from previous studies ${ }^{3}$ 
Table 2. Dependence of reaction parameters on the shape of wood samples

\begin{tabular}{|c|c|c|c|c|c|c|}
\hline Sample & Temperature & Ultimate weight gain (\%) & $k\left(\mathrm{~h}^{-1}\right)$ & $n$ & No. of data & $R^{2}$ \\
\hline \multirow[t]{2}{*}{$\operatorname{Meal}^{\mathrm{a}}(150-355 \mu \mathrm{m})$} & $100^{\circ} \mathrm{C}$ & 28.8 & $3.70 \times 10^{-2}$ & 1.28 & 14 & 0.990 \\
\hline & $120^{\circ} \mathrm{C}$ & 26.4 & $3.79 \times 10^{-1}$ & 1.40 & 14 & 0.977 \\
\hline \multirow[t]{3}{*}{ Meal $(355-850 \mu \mathrm{m})$} & $100^{\circ} \mathrm{C}$ & 26.0 & $9.45 \times 10^{-2}$ & 0.75 & 6 & 0.935 \\
\hline & $110^{\circ} \mathrm{C}$ & 29.0 & $1.15 \times 10^{-1}$ & 1.13 & 8 & 0.979 \\
\hline & $120^{\circ} \mathrm{C}$ & 29.5 & $2.47 \times 10^{-1}$ & 1.70 & 8 & 0.969 \\
\hline \multirow[t]{3}{*}{ 2-mm block } & $100^{\circ} \mathrm{C}$ & 22.5 & $2.96 \times 10^{-2}$ & 3.96 & 6 & 0.977 \\
\hline & $110^{\circ} \mathrm{C}$ & 23.3 & $1.31 \times 10^{-1}$ & 3.33 & 8 & 0.940 \\
\hline & $120^{\circ} \mathrm{C}$ & 21.3 & $3.48 \times 10^{-1}$ & 2.55 & 7 & 0.997 \\
\hline 5-mm block ${ }^{\mathrm{b}}$ & $120^{\circ} \mathrm{C}$ & 21.3 & $6.36 \times 10^{-1}$ & 1.76 & 8 & 0.968 \\
\hline
\end{tabular}

The reaction was carried out without a catalyst

${ }^{a}$ Data from previous studies ${ }^{3}$

${ }^{\mathrm{b}}$ Recalculated based on data from previous studies ${ }^{3}$

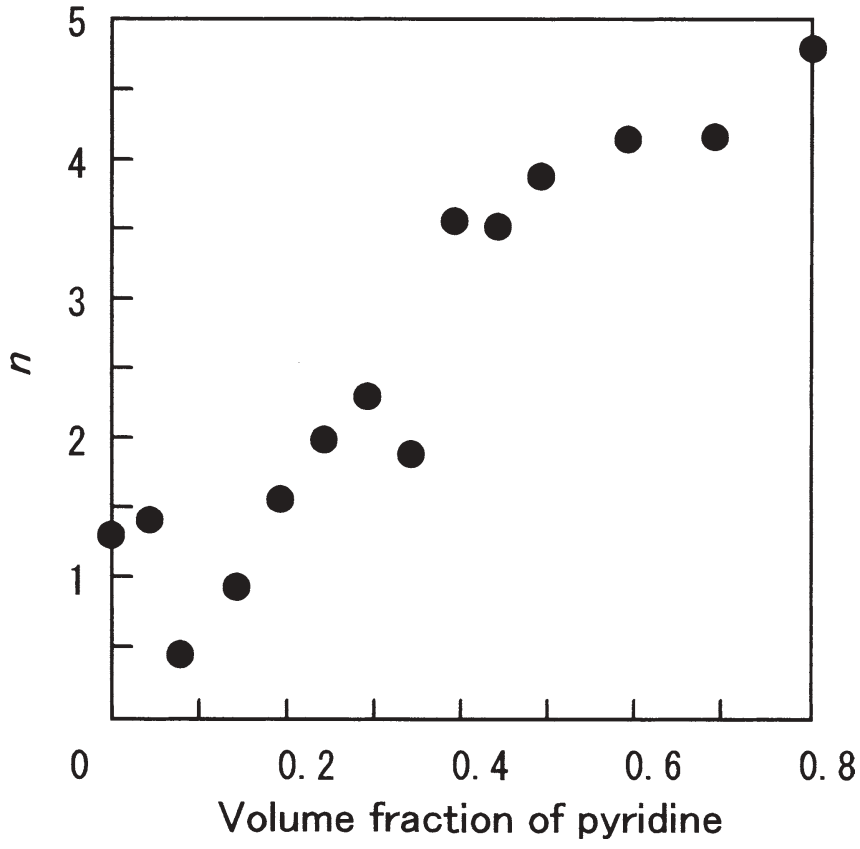

Fig. 5. Dependence of the parameter $n$ (hindrance against diffusion of reagent to reaction site) on the volume fraction of pyridine in the pyridine-acetic anhydride mixtures

Table 1 includes the result obtained for the DMFcatalyzed acetylation. The $k$ value in the DMF-acetic anhydride system ( $\mathrm{v} / \mathrm{v}, 2: 8)$ was about three times larger than that for the uncatalyzed reaction but less than one tenth of the value for the pyridine-acetic anhydride system $(\mathrm{v} / \mathrm{v}$, $2: 8)$ at $100^{\circ} \mathrm{C}$. Because the swelling in the presence of DMF did not differ greatly from that in the presence of pyridine, the difference is attributable to the strength of catalysis.

Dependence of reaction parameters on the dimensions of the sample

Equation 1 was also applied to the data obtained for wood samples of various dimensions. The reaction parameters and statistical data are summarized in Table 2. The applicability of the rate equation was good for all reaction conditions.

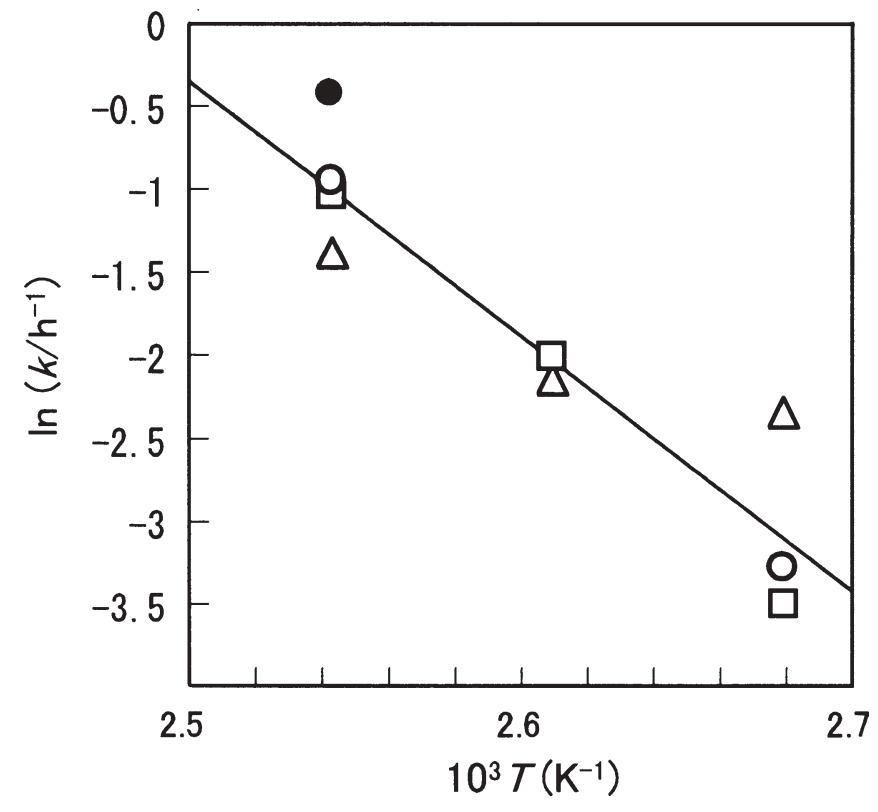

Fig. 6. Arrhenius plot and regression line adjusted to whole plots. Open circles, fine wood meal; triangles, coarse wood meal; squares, 2$\mathrm{mm}$ cross section; filled circle, 5 -mm cross section

Figure 6 shows a so-called Arrhenius plot. The $k$ value did not significantly differ with the dimensions or shapes of the samples, although it depended on the reaction temperature. This result contradicts the one reported previously. ${ }^{3}$ By introducing the parameter $n$, the influence of diffusion upon the rate constant was canceled, and a rate constant closer to the real value could be obtained. The activation energies estimated from the slope of the Arrhenius plot were about 140,60 , and $150 \mathrm{~kJ} / \mathrm{mol}$ for fine wood meal, coarse wood meal, and 2-mm-thick wood blocks, respectively. However, we cannot explain why the activation energy for the coarse wood meal was lower than the other samples. It is possible that the low value observed for coarse wood meal may be accidental. When the activation energy was calculated irrespective of the type of sample, a value of about $130 \mathrm{~kJ} / \mathrm{mol}$ was obtained from the regression line drawn in Fig. 6, which was much greater than that reported by Hill et al. ${ }^{1}$ 
The $n$ value was significantly larger for the cross sections than for wood meal, irrespective of the reaction temperature. This means that the reaction rate for the cross sections is strongly controlled by the diffusion of reagent. Parameter $n$ seems to change critically between wood meal and the 2 mm cross sections; however, because the mean length of the softwood tracheid is thought to be about $3-4 \mathrm{~mm}$, it is unlikely that the probability of cutting tracheids increases suddenly at around $1 \mathrm{~mm}$. The reasons why $n$ differs between wood meal and the cross sections may be (1) the wood fibers are bound together in the wood block, and the bundle interferes with the swelling of each fiber, whereas wood meal can swell without such a restraint, and (2) in wood meal, the damage undergone during milling may facilitate the diffusion of reagent into the cell wall.

The ultimate weight gain of a cross section was a few percent lower than that of wood meal. This may also be explained by the two reasons given above.

\section{Conclusions}

Factors influencing the rate of acetylation were examined based on swelling in a reaction solution and the dimensions of wood samples. The results can be summarized as follows:

1. Above $60^{\circ} \mathrm{C}$, at which temperature acetylation is normally carried out, wood swells thoroughly, even in neat acetic anhydride. Therefore, pyridine may facilitate the reaction as a catalyst rather than as a swelling agent.
2. In the pyridine-acetic anhydride system, the optimum volume fraction of pyridine was found to be around 0.2 , based on the reaction kinetics.

3. With the increase in pyridine content, the reaction took on a diffusion-controlled character.

4. By applying a modified rate equation, we could estimate the reaction rate, which excluded the effect of the shape of the wood sample, and obtained an activation energy of about $130 \mathrm{~kJ} / \mathrm{mol}$. Therefore, we believe that the activation energy of acetylation is not as small as that proposed by Hill et al. ${ }^{1}$ after removal of the influence of diffusion.

\section{References}

1. Hill CAS, Jones D, Strickland G, Cetin NS (1998) Kinetic and mechanistic aspects of the acetylation of wood with acetic anhydride. Holzforschung 52:623-629

2. Hill CAS, Papadopoulos AN (2002) The pyridine-catalysed acetylation of pine sapwood and phenolic model compounds with carboxylic acid anhydrides. Determination of activation energies and entropy of activation. Holzforschung 56:150-156

3. Minato K, Ogura K (2003) Dependence of reaction kinetics and physical and mechanical properties on the reaction systems of acetylation I: reaction kinetic aspects. J Wood Sci 49:418-422

4. Ishimaru Y, Yamada Y, Iida I, Urakami H (1996) Dynamic viscoelastic properties of wood in various stages of swelling (in Japanese). Mokuzai Gakkaishi 42:250-257

5. Minato K (2004) Application of a modified rate equation to the acetylation of wood. J Wood Sci (in press)

6. Stamm AJ (1964) Wood and cellulose science. Ronald, New York, p 330 